\title{
Community pharmacy practice and challenges amid the coronavirus disease-19 pandemic: A survey in southwest Ethiopia
}

\author{
Behailu TEREFE 1 * (D), Mengist AWOKE 1 (D), Dula DESSALEGN ${ }^{1}$ (D), Girma MAMO 1 (D), \\ Bezie KEBEDE ${ }^{2}$ (D), Temesgen MULUGETA ${ }^{1}$ (i) \\ 1 School of Pharmacy, Faculty of Health Sciences, Jimma University, Po.Box:378, Jimma, Ethiopia. \\ 2 School of Pharmacy, College of Health Sciences, Mizan-Tepi University, Mizan-Aman, Ethiopia. \\ * Corresponding Author. Email: terefebh@gmail.com (B.T); Tel. +25-192-019 4883.
}

Received: 28 April 2021 / Revised: 08 September 2021 / Accepted: 02 November 2021

\begin{abstract}
The coronavirus disease-19 (COVID-19) pandemic has challenged and forced the community pharmacies across the world to take various measures to prevent its spread. This survey was conducted to assess the community pharmacy practice and challenges in Ethiopia amid the coronavirus disease-19 pandemic. The survey was conducted involving 67 community pharmacy professionals in Jimma and Mizan-Aman towns in the period from November 25 to December 10, 2020. A checklist was used for the data collection, and descriptive analysis was conducted using SPSS V.22. In this study, druggists were higher in proportion than pharmacists (64.2\% vs. $35.8 \%)$. Of the stock of the COVID19 infection protection supplies, facemask was well-stocked (73.1\%), whereas most respondents $(73.1 \%)$ reported no stock of face shields. Practicing a distance of $>1$ meter from the customers $(85.1 \%)$ and keeping hand hygiene $(83.6 \%)$ were among the measures reported to prevent cross-infection. Above two-third of the respondents reported an increase in verbal queries of the COVID-19 information (68.7\%). Financial implication $(89.6 \%)$ and staff cross-infection risk $(89.6 \%)$ were the major worries reported by the respondents. In this study, some of the sociodemographic information were identified to affect the COVID-19 precautionary measures and challenges. In conclusion, the present study revealed problem with availability of some of the COVID-19 prevention supplies, like face shields (73.1\%), requiring effort to avail. An increase in COVID-19 related queries from customers was also reported. The COVID-19 precautionary measures, such as practicing hand hygiene, physical distancing, and others were reported. The reported financial and other challenges of the COVID-19 needs mitigation measures.
\end{abstract}

KEYWORDS: COVID-19; community pharmacy; practice; challenge; pharmacy professionals; Ethiopia.

\section{INTRODUCTION}

The COVID-19 pandemic has still remained as the most important challenges of the globe. The cases and associated deaths are increasing from time to time in some countries, such as Ethiopia [1]. In the early phase, with the rampant spread of the COVID-19 pandemic, various health organizations, institutions, and governments worldwide have taken several measures to reduce the spread of the disease. These measures included hand and environmental hygiene techniques, physical distancing, travel restrictions, border control, quarantine and lockdown measures, and many more [2].

Community pharmacies, like the rest of society and health institutions, struggled to cope but learned important lessons for whatever comes next with this pandemic [3]. Because of the spread of this pandemic disease, worldwide, pharmacists had been changing their roles and being increasingly recognized for their role as essential service providers ranging from supplying essential medicines and equipment to extended responsibilities of emergency care [4]. They remained frontline providing the public health service. Their practice has had to adapt significantly, but the pandemic has also focused attention on the case for longawaited professional role evolution as direct access for the patients especially while there is an outbreak [5]. In Saudi Arabia, pharmacists contributed many to protect the public from COVID-19 by participating in various initiatives including health education and promotion, medication dispensing and reconciliation, patient counseling, information for self-management in the current outbreak, and emergency preparedness [6].

How to cite this article: Terefe B, Awoke M, Dessalegn D, Mamo G, Kebede B, Mulugeta T. Community pharmacy practice and challenges amid the coronavirus disease-19 pandemic: A survey in southwest Ethiopia. J Res Pharm. 2021; 25(6): 890-897. 
The COVID-19 pandemic has posed serious challenges to the health systems, straining and overwhelming healthcare facilities including hospitals [7] and community pharmacy pharmaceutical care services [8]. During the outbreak, community pharmacists were faced with several challenges including, an increasing number of people visiting the community pharmacies; many are unable to access their physicians for a recheck because of their frustration of acquiring the disease from the hospital upon visiting. This means that the only health care professional available to many people and patients turned up to be community pharmacies even in serious conditions. The other immediate challenge was protecting their staff and patients from the spread of infection within the pharmacy. Pharmacies adapted their premises to try and achieve 'social distancing, installing Perspex barriers or doorway booths, restricting. Although COVID-19 has been a very challenging and stressful period for community pharmacy, the government and the community recognized it as a front-line and essential. The necessity of crisis has led to the expansion of professional roles, responsibilities, and significant adaptation to models of care. This recognition and professional roles improve pharmacist appetite for further role expansion in the community [9].

There are no sufficient studies even world-wide and surprisingly enough, in Ethiopia, only limited studies are done concerning COVID-19 related preparedness and knowledge and attitude [10, 11]. The challenges were not discovered yet. Therefore, there is a need to identify the challenges of community pharmacy and forward what should be done next. The primary aim of this study was (i) to explore the practice of community pharmacy and (ii) to identify challenges faced amid the outbreak.

\section{RESULTS}

\subsection{Overview of the study}

In this study, a total of 67 CPPs from 49 community drug retail outlets completed the survey format (response rate $=85.9 \%$ ) and were included in the analysis (Figure 1 ).

\subsection{Socio-demographic characteristics of the participants}

The median age of the respondents was $31(\mathrm{IQR}=8)$ years, and above two-third $(\mathrm{n}=45 ; 67.2 \%)$ were male. Of the total respondents, owners of the drug outlet accounted for about half $(n=34 ; 50.7 \%)$. The professionals were predominantly druggists $(n=43 ; 64.2 \%)$. The median years of experience of the professionals was 7 $(\mathrm{IQR}=6)$ years (Table 1$)$.

\subsection{The self-reported stock of COVID-19 prevention supplies}

All of the respondents reported the availability of facemasks in their settings. They reported that it is well-stocked supply $(n=49 ; 73.1 \%)$ followed by sanitizers $(n=48 ; 71.6 \%)$. Most of the respondents reported no stock of face shields $(\mathrm{n}=49 ; 73.1 \%)$ (Table 2$)$.

Table 1. Information on the socio-demographic characteristics of the respondents and community settings approached in Jimma and Mizan-Aman towns, November 25 to December 10, 2020 (N=67).

\begin{tabular}{lcc}
\hline Socio-demographic characteristics & & \\
\hline Age in years, Median (IQR) & & $31(8)$ \\
Sex & Male & $45(67.2 \%)$ \\
& Female & $22(32.8 \%)$ \\
Marital status & Single & $23(34.3 \%)$ \\
& Married & $39(58.2 \%)$ \\
Having child & Divorced & $5(7.5 \%)$ \\
& Yes & $33(49.3 \%)$ \\
Professionals`experience in years, Median (IQ) & No & $34(50.7 \%)$ \\
Ownership & & $7(6)$ \\
& Owner & $34(50.7 \%)$ \\
Professional level & Not owner & $33(49.3 \%)$ \\
& Pharmacist & $24(35.8 \%)$ \\
Setting type & Druggist & $43(64.2 \%)$ \\
& Pharmacy & $16(32.7 \%)$ \\
& Drug store & $33(67.3 \%)$ \\
\hline
\end{tabular}

IQR-Interquartile range 


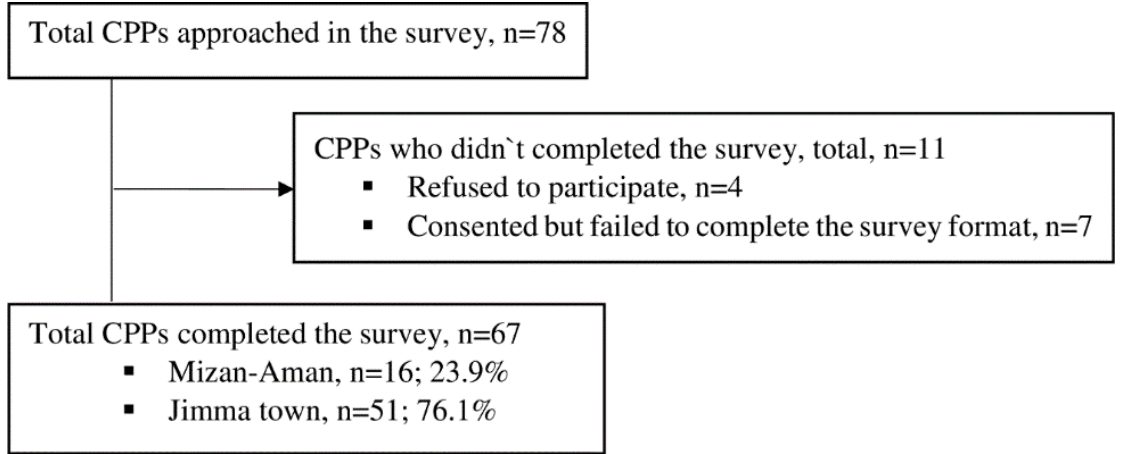

Figure 1. Flow chart showing an overview of the study. CPPs-Community Pharmacy Practitioners`.

Table 2. Stock of COVID-19 prevention equipment and sanitizer in the community pharmacy settings in Jimma and Mizan-Aman towns, November 25 to December 10, 2020 (N=67).

\begin{tabular}{llll}
\hline Supplies & Well stocked, $\mathbf{n}(\mathbf{\%})$ & Limited stock, $\mathbf{n}(\mathbf{\%})$ & No stock, $\mathbf{n}(\mathbf{\%})$ \\
\hline Face mask & $49(73.1)$ & $18(26.9)$ & 0 \\
Hand glove & $32(47.8)$ & $24(35.8)$ & $11(16.4)$ \\
Face shield & $10(14.9)$ & $8(11.9)$ & $49(73.1)$ \\
Hand sanitizer & $48(71.6)$ & $16(23.9)$ & $3(4.5)$ \\
\hline \multicolumn{2}{c}{ COVID-19-Coronavirus disease-19 }
\end{tabular}

\subsection{Changes in the pharmacy work setting, practice, and logistic procedures}

Most respondents reported to follow precautionary measures to prevent the COVID-19 cross-infection, such as practicing a physical distance of $>1 \mathrm{~m}$ from the customers $(\mathrm{n}=57 ; 85.1 \%)$, using hand sanitizer/washing hand after each transaction $(n=56,83.6 \%)$, keeping water with soap at the entrance of the setting for customers $(\mathrm{n}=55 ; 82.1 \%)$, and keeping a physical distance of $>1 \mathrm{~m}$ among customers $(\mathrm{n}=53 ; 79.1 \%)$ and among the staffs $(\mathrm{n}=52 ; 77.6 \%)$. The service of prescription deliveries to patient`s homes accounted for the lowest proportion $(\mathrm{n}=4 ; 6.0 \%)$ (Table 3$)$.

Table 3. Changes in the pharmacy work setting and logistics to contain the spread of COVID-19 in Jimma and Mizan-Aman towns, November 25 to December 10, 2020 (N=67).

\begin{tabular}{lll}
\hline Changes in the pharmacy work setting and logistic procedures & Yes, n (\%) & No, n (\%) \\
\hline Practice social distance among staffs and customers (>1m) & $57(85.1)$ & $10(14.9)$ \\
Use of hand sanitizer or washing hand after each transaction & $56(83.6)$ & $11(16.4)$ \\
Keeping water with soap at the entrance of the setting & $55(82.1)$ & $12(17.9)$ \\
Practice social distance among customers (>1m) & $53(79.1)$ & $14(20.9)$ \\
Practice social distance among staffs (>1m) & $52(77.6)$ & $15(22.4)$ \\
Taping or marking distance lines on the floor & $49(73.1)$ & $18(26.9)$ \\
Regular cleaning and disinfection of the working area & $49(73.1)$ & $18(26.9)$ \\
Change in pharmacy/drug store opening status during the COVID-19 outbreak & $47(55.2)$ & $30(44.8)$ \\
Staffs wearing facemask & $46(68.7)$ & $21(31.3)$ \\
keeping hand sanitizers at the entrance of the setting for the customers & $42(62.7)$ & $25(37.3)$ \\
Posting public educational posters on the signs, symptoms, and prevention of COVID-19 & $40(59.7)$ & $27(40.3)$ \\
Restriction of customer numbers in the Pharmacy & $32(47.8)$ & $35(52.2)$ \\
Breaking staffs into teams to avoid COVID-19 cross-infection & $26(38.8)$ & $41(61.2)$ \\
Staffs wearing glove & $21(31.3)$ & $46(68.7)$ \\
Installation of glass or plastic barrier & $20(29.9)$ & $47(70.1)$ \\
Change of service from door to window & $19(28.4)$ & $48(71.6)$ \\
Installation of doorway booth & $17(25.4)$ & $50(74.6)$ \\
Shortened working hours after the COVID-19 outbreak & $16(23.9)$ & $51(76.1)$ \\
Staffs wearing a face shield & $13(19.4)$ & $54(80.6)$ \\
Prescription deliveries at home & $4(6.0)$ & $63(94.0)$
\end{tabular}

COVID-19-Coronavirus disease-19, m-meter 
In above two-third $(n=46 ; 68.7 \%)$ of the professionals, an increase in the number of customers with verbal queries of the COVID-19was reported, but only less than half of the total respondents $(n=27 ; 40.3 \%)$ reported an overall increase in drug information query after the COVID-19 outbreak. After the outbreak, an increase in off-prescription medication request $(\mathrm{n}=27 ; 40.3 \%)$ and request of medications on medical complaint $(\mathrm{n}=18 ; 26.9 \%)$ were reported by the respondents. Likewise, less than a quarter of respondents reported an increase in medication refill queries $(\mathrm{n}=10 ; 14.9 \%)$. A little above quarter of respondents reported an increase in the customer waiting time $(n=18 ; 26.9 \%)$ due to the COVID-19 related measures. The respondents predominantly reported television as the source of COVID-19 information $(n=48 ; 71.6 \%)$ followed by social media $(n=44 ; 65.7 \%)$ and various websites $(n=38 ; 56.7 \%)$ (Figure 2$)$.

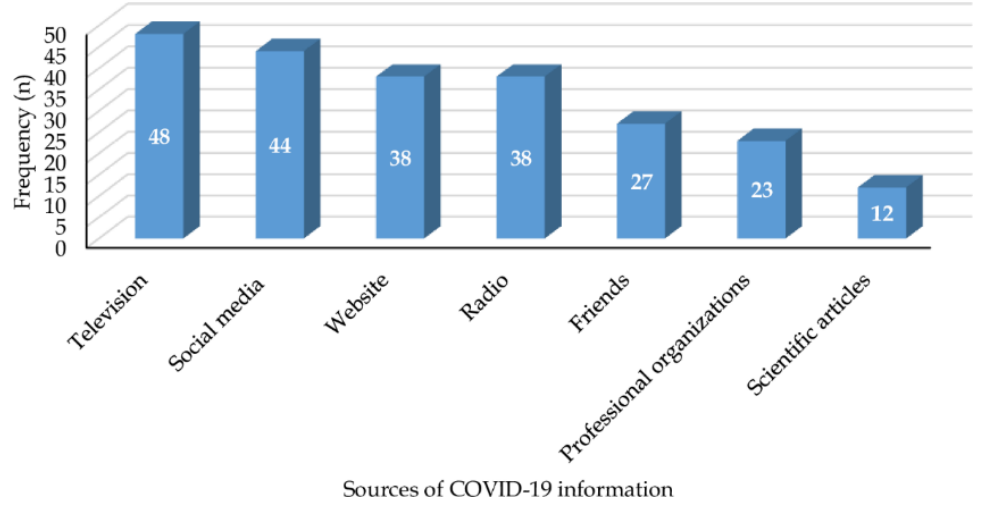

Figure 2. Potential source of COVID-19 information. The source of COVID-19 information used by the CPPs is presented in the descending order of frequency from left to right. CPPs- Community Pharmacy practitioners'.

\subsection{Challenges to community pharmacy practice during the COVID-19 pandemic}

The CPPs had sustained various challenges (worries) after the COVID-19 outbreak. Most of the respondents $(n=46 ; 68.7 \%)$ declared an increased worrisome feeling due to increased frequency of regulatory inspection from the Ethiopian Food, Medicine and Healthcare Administration and Control Authority (EFMHACA) after the COVID-19 outbreak. Worry of the financial implication of the COVID-19 outbreak $(n=60 ; 89.6 \%)$ and getting infected oneself and fellow staffs with COVID-19 $(n=60 ; 89.6 \%)$ were the upper hand challenges reported by the respondents (Table 4 ).

Table 4. Challenges posed by the COVID-19 outbreak on the community pharmacy practitioners in Jimma and Mizan-Aman towns, November 25 to December 10, 2020 (N=67).

\begin{tabular}{lcc}
\hline Challenges & Increased & $\begin{array}{c}\text { Not } \\
\text { increased }\end{array}$ \\
\hline Worry about the financial implication of the COVID-19 outbreak & $60(89.6)$ & $7(10.4)$ \\
Worry of getting infected oneself and fellow staffs with COVID-19 & $60(89.6)$ & $7(10.4)$ \\
Worry of risk of infecting loved ones & $54(80.6)$ & $13(19.4)$ \\
Worry of the restriction of the normal adaptive coping strategies & $54(80.6)$ & $13(19.4)$ \\
Worry about drug shortage after COVID-19 outbreak & $53(79.1)$ & $14(20.9)$ \\
Worry of the frequent regulatory inspection & $46(68.7)$ & $21(31.3)$ \\
Worry of misinformation about the COVID-19 and related issues & $45(67.2)$ & $22(32.8)$ \\
Worry about my mental health after the COVID-19 outbreak & $38(56.7)$ & $29(43.3)$ \\
Worry of inadequate COVID-19 information & $37(55.2)$ & $30(44.9)$ \\
Verbal abuse after the COVID-19 outbreak & $36(53.7)$ & $31(46.3)$ \\
Worry of lack of child care after the COVID-19 outbreak & $33(49.3)$ & $34(50.7)$ \\
Worry about workload / staff shortage after COVID-19 outbreak & $31(46.3)$ & $36(53.7)$ \\
\hline \multicolumn{2}{c}{ COVID-19-Coronavirus disease-19 } &
\end{tabular}

\subsection{Distribution of responses to the adapted precautionary measures, psychological and other challenges of the respondents after the COVID-19 outbreak in relation to their sociodemographic information}

In this study, those professionals who reported installation of glass or plastic barrier in dispensaries to prevent the transmission of COVID-19 have a higher work experience in years $(8 ; \mathrm{IQR}=5$ vs. 6 ; IQR=5, $\mathrm{p}<0.001)$ and have no child ( $\mathrm{n}=15 ; 75.0 \%$ vs. $\mathrm{n}=5 ; 25.0 \%, \mathrm{p}=0.01$ ) as compared to those who reported no installation. Additionally, as compared to pharmacists, higher proportion of druggists reported a regular cleaning and 
disinfection of their work setting $(\mathrm{n}=28 ; 57.1 \%$ vs. $\mathrm{n}=21 ; 42.9 \%, \mathrm{p}=0.04)$ as a precautionary measures against the COVID-19 transmission.

With regard to the psychological and other challenges after the COVID-19 outbreak, a higher proportion of those professionals who reported an increase in worrisome feeling from increased workload and staff shortage were predominantly males (males, $\mathrm{n}=17 ; 54.8 \%$ vs. females, $\mathrm{n}=14 ; 45.2 \%, \mathrm{p}=0.04$ ); younger in age (median age in years, $31 ; \mathrm{IQR}=4$ vs. $32 ; \mathrm{IQR}=9, \mathrm{p}=0.02)$; and had less work experience in years (6; IQR=5, vs. 8.5; $\mathrm{IQR}=7.3, \mathrm{p}=0.02$ ) as compared to those who reported no change in the feeling. Furthermore, compared to pharmacists, higher proportion of druggists reported an increase in worrisome feelings due to drug shortage after the COVID-19 outbreak ( $\mathrm{n}=30 ; 56.6 \%$ vs. $\mathrm{n}=23 ; 43.4 \%, \mathrm{p}=0.01$ ). On the other hand, those professionals who have no child were more frequently reported an increase in verbal abuse from customers $(n=24 ; 66.7 \%$ vs. $\mathrm{n}=12 ; 33.3 \%$; $\mathrm{p}=0.01)$; an increase in worrisome feeling due to increased frequency of regulatory inspection of the dispensary $(\mathrm{n}=28 ; 60.9 \%$ vs. $\mathrm{n}=18 ; 39.1 \%, \mathrm{p}=0.01)$, and an increase in worrisome feeling due to personal lack of adequate COVID-19 information ( $\mathrm{n}=23 ; 62.2 \%$ vs. $\mathrm{n}=14 ; 37.8 \%$, $\mathrm{p}=0.03)$.

\section{DISCUSSION}

During the present survey period, most of the community pharmacies had a well-stocked facemask (73.1\%) and sanitizers (71.16\%). A study done in Egypt reported comparable findings, where facemasks were available in $75.6 \%$ of community pharmacies; while sanitizers other than alcohol were available in $86.9 \%$ of the community pharmacies [12]. In contrast, most of the pharmacies (73.1\%) do not have the stock of face shields. Which might imply poor supply chain management in the settings.

Regarding the precautionary measures to contain the virus, $79.1 \%$ of the CPPs surveyed practiced a safe physical distancing of at least $1 \mathrm{~m}$ between the customers. And only $47.8 \%$ of the community pharmacies restrict the number of customers in the pharmacies. These precautionary measures taken were lower compared to a study done in the United Kingdom (UK), where $84.0 \%$ of the pharmacists maintained a safe distance of $2 \mathrm{~m}$ from customers and $80.6 \%$ of them limited the number of customers in a given time to maintain social distancing [13]. In Egypt, the physical distance of at least $1 \mathrm{~m}$ was practiced in $95.5 \%$ among the personnel at the community pharmacy. Similarly, the prevention of customer crowding was implemented in $94.7 \%$ of community pharmacies [12].

In this study, $68.7 \%$ of the pharmacists reported wearing a facemask. In the UK, relatively a higher number of pharmacists $(72 \%)$ used surgical or N95 for protection. Moreover, most $(70.1 \%)$ of the surveyed pharmacies were not installed with plastic barriers to contain the virus compared to $75.7 \%$ of the pharmacies in the UK that installed a plastic barrier. Hand sanitizers were placed at the entrance in $62.7 \%$ of pharmacies to be used by customers for sanitizing their hands before entering. This measure was more reported in our study compared to similar studies from the UK (24.8\%) [13] and Australia (35.77\%) [14]. The prescriptions that pass through the hands of the patients, doctors, and nurses are a potential source of pathogens, putting the community pharmacy personnel at risk [15]. In this study, the practice of wearing gloves in the community pharmacy was very low (31.3\%), however, most of them used hand sanitizer after each transaction (83.6\%). These practices were low compared to a study done in Kosovo, where $97 \%$ of the pharmacists used protective gloves and hand disinfectants $(96.2 \%)$ followed by surgical masks $(81.1 \%)$, respectively during their practice [16]. This is because, in this study, $16.4 \%$ of protective gloves were reported to be not stocked in the community pharmacies. Forty pharmacies $(59.7 \%)$ were claimed to post public educational posters about signs and symptoms of COVID-19 infection and prevention at the entrance of pharmacies. However, $75 \%$ of the pharmacies in the UK had visible posters at the entrance [13].

Disease characteristics, organizational factors, and personal factors may contribute to developing psychological distress and other mental health symptoms [17]. In this survey, most (89.6\%) of the CPPs were worried about the risk of being self-infected and infecting fellow staff with the virus, and the financial implication of the pandemic. Similarly, a study reported that $90 \%$ of the pharmacy professionals reported being at least somewhat worried and over $65 \%$ were very worried or extremely about the impact of COVID19 on them personally and professionally [18]. Our finding showed, $56.7 \%$ of the CPPs were worrying about their mental health after the outbreak. In France, up to $35 \%$ of pharmacists reported psychological disturbances (17\% reported significant post-traumatic stress symptoms) [19]. Even though this survey did not test CPPs for the virus, a study reported that $14.6 \%$ of the community pharmacy personnel were tested positive for antibody which could put them stressed [20].

Community pharmacists are essential contributors to public health and play a key role to combat COVID-19 [21]. In this survey, the COVID-19 and related information query was increased by $68.7 \%$ during 
the pandemic. In Poland, the majority of the pharmacists and pharmacy technicians provided an evidencebased recommendation on prevention, symptoms, and management of SARS-CoV-2 up on the mysterious call [22]. In this study, after the disease outbreak, social distancing increased the staff shortage and workload by $68.7 \%$ and $56.7 \%$, respectively. However, a lower response was reported somewhere that, $28 \%$ of respondents stated social distancing increased workload and visits to the pharmacy [18]. The reason for the discrepancy might be due to the difference in the number of pharmacy professionals allocated to the community pharmacies and the sample size of the respondents.

In this survey, the majority of the CPPs got the COVID-19 related information from television $(71.6 \%)$ and social media $(65.7 \%)$. This practice in contrast to a study done in Kosovo, where information from professional organizations $(85.2 \%)$ and internet websites $(73.1 \%)$ was the main source used by community pharmacists [16]. In our settings, a few community pharmacists were used scientific articles (17.9\%). However, a study from Kosovo showed $51.9 \%$ of the community pharmacists reported the use of scientific articles for the COVID-19 related information [16]. The discrepancy might be related to the difference in the issues coming with the study settings.

In this study, a more frequent reports of implementing some of the COVID-19 precautionary measures were identified from druggist professionals, professionals with a higher work experience, and those who have no child as compared to their counterparts. On the other hand, professionals who are druggists, male in sex, younger in age, who have no child, and with less work experience had frequently reported some of the psychological and other challenges after the COVID-19 outbreak as compared to the others.

\section{CONCLUSION}

The present study revealed a lack of availability of some of the COVID-19 prevention supplies in the settings which need the effort to ensure adequacy. An increase in COVID-19 related queries from customers was reported during the pandemic. Hand hygiene, physical distancing, and some premises-related changes that help to prevent the COVID-19 infection had also been reported. Worries on the economic and psychological impact of the COVID-19 had also been reported. Thus, strategies to mitigate the economic and psychological impact of the COVID-19 are imperative measures to ensure the existence and continuation of these essential settings. Some of the sociodemographic information were identified to affect the COVID-19 precautionary measures and challenges faced by the professionals. But, due to the small sample size, the authors of this study expresses their reservation of confidently concluding the effect.

\section{MATERIALS AND METHODS}

\subsection{Study design and setting}

A cross-sectional survey was conducted at Jimma and Mizan-Aman towns by approaching community Pharmacy professionals (CPPs) practicing in the private and public community pharmacies and drug stores from November 25 to December 10, 2020. Jimma and Mizan-Aman towns are located in south-west Ethiopia, $346 \mathrm{~km}$ and $581 \mathrm{~km}$ away from Addis Ababa, respectively.

\subsection{Sample size and sampling technique}

All consented CPPs (Pharmacists and Druggists) $(\mathrm{N}=67)$ were included in this study using purposive sampling technique.

\subsection{Study variables}

Demographic profile (age, sex, working experience, working site, Pharmacy/drug store ownership, number of working staff), personal protective equipment and supplies (face mask, glove, sanitizer, face shields), implementation of safety measures (installing glass/plastic screen barriers, taping/marking 'distance' lines on the floor, restricting patient numbers for access, installing doorway booth, wearing face shields, and others), pharmacists' concerns during COVID-19 and harassment of pharmacy staff since the COVID-19 were considered as study variables.

\subsection{Data collection instrument and process}

The data collection tool was developed extracting relevant variables from various literature. The tool had four parts: 1) Socio-demographic characteristics of the participants, 2) Self-reported stock of COVID-19 prevention supplies, 3) Changes in the pharmacy work setting, practice, and logistic procedures, 4) Challenges 
to the community pharmacy practice during the COVID-19 pandemic (Pharmacists' concerns and sources of information during COVID-19, harassment/abuse of pharmacy staff since COVID-19 outbreak). Four data collectors (Bachelor's degree in Pharmacy) and two supervisors (Master's degree in Clinical Pharmacy) were employed. The data collectors and supervisors were made familiar with the objectives of the study, the data collection tool, and procedures. The data collectors then approached the community pharmacies and drug stores located in the two towns to access CPPs and requested informed consent with the intent to confirm their willingness to participate. Then, consented professionals were given the survey format, and the data collectors completed the distribution of the checklists within the fifth day. Each participant was told to complete the format within four working days beginning from the day they received the format. On the fifth day, the data collectors approached each participant for collecting the filled formats. Some of the participants were given an additional one day to complete filling of the format when they failed to do so on the fifth day.

\subsection{Data analysis}

Data entry and analysis were conducted using SPSS V.22. Categorical variables were described with frequency and percentage, while median with interquartile range (IQR) was calculated for continuous variables. Statistical comparisons between groups were made using the chi-square $\left(\chi^{2}\right)$ test (categorical variables) and Mann-Whitney U test or Kruskal-Wallis test (continuous variables). For all statistics, a pvalue $<0.05$ was considered as a threshold to declare statistical significance.

\subsection{Definition of terms}

Druggists are pharmacy professionals' who hold a diploma in pharmacy, whereas pharmacists are pharmacy professionals' who have a bachelor's degree in pharmacy.

Acknowledgements: We would like to thank Jimma University for evaluating and approving the protocol of this study. Our gratitude also extends to the CPPs who kindly showed their cooperation to participate in the study.

Author contributions: Concept- B.T, M.A.; Design- B.T, M.A, B.K, G.M.; Supervision- B.T, M.A, G.M., B.K.; Resource and Materials- B.T, M.A, G.M., B.K.; Data Collection \&/or Processing- B.T, M.A, D.D., B.K.; Analysis \&/or Interpretation- B.T, M.A, T.M.; Literature Search- B.T, M.A, D.D, G.M, B.K, and T.M.; Writing manuscript-B.T.; Critical Reviews- B.T, M.A, D.D, G.M, B.K, and T.M.

Conflict of interest statement: The authors declared no conflict of interest.

Ethics committee approval and consent to participate: The study protocol was ethically approved by the institutional review board (IRB) of Jimma University (Ref.no: IHRGD/996/20). Participants were approached with a formal request for written consent to participate. All raw data collected from the participants were used only for this study purpose.

\section{REFERENCES}

[1] WHO Coronavirus (COVID-19) Dashboard 2021. https://covid19.who.int/, (accessed on 22 May 2021).

[2] Hoti K, Jakupi A, Hetemi D, Raka D, Hughes J, Desselle S. Provision of community pharmacy services during COVID 19 pandemic: a cross sectional study of community pharmacists' experiences with preventative measures and sources of information. Int J Clin Pharm. 2020; 42(4): 1197-1206. [CrossRef]

[3] Gregory PAM, Austin Z. COVID-19: How did community pharmacies get through the first wave? Can Pharm J (Ott). 2020; 153(5): 243-251. [CrossRef]

[4] Alves da Costa F, Lee V, Leite SN, Murillo MD, Menge T, Antoniou S. Pharmacists reinventing their roles to effectively respond to COVID-19: a global report from the international pharmacists for anticoagulation care taskforce (iPACT). J Pharm Policy Pract. 2020; 13(1): 1-3. [CrossRef]

[5] Bukhari N, Rasheed H, Nayyer B, Babar Z-U-D. Pharmacists at the frontline beating the COVID-19 pandemic. J Pharm Policy Pract. 2020; 13(1): 1-4. [CrossRef]

[6] Ahmad A, Alkharfy KM, Alrabiah Z, Alhossan A. Saudi Arabia, pharmacists and COVID-19 pandemic. J Pharm Policy Pract. 2020;13: 41.[CrossRef]

[7] Bragazzi NL, Mansour M, Bonsignore A, Ciliberti R. The Role of Hospital and Community Pharmacists in the Management of COVID-19: Towards an Expanded Definition of the Roles, Responsibilities, and Duties of the Pharmacist. Pharmacy (Basel, Switzerland). 2020; 8(3): 1-15. [CrossRef] 
[8] Hamed SH. Community Pharmacy Practice During COVID-19 Pandemic: A Perspective From the Middle East. JQSH. 2020; 3(3): 109-114. [CrossRef]

[9] Hayden JC, Parkin R. The challenges of COVID-19 for community pharmacists and opportunities for the future. Ir J Psychol Med. 2020; 37(3): 198-203. [CrossRef]

[10] Kasahun GG, Kahsay GM, Asayehegn AT, Demoz GT, Desta DM, Gebretekle GB. Pharmacy preparedness and response for the prevention and control of coronavirus disease (COVID-19) in Aksum, Ethiopia; a qualitative exploration. BMC Health Serv Res. 2020; 20(1): 913: 1-6. [CrossRef]

[11] Tesfaye ZT, Yismaw MB, Negash Z, Ayele AG. COVID-19-Related Knowledge, Attitude and Practice Among Hospital and Community Pharmacists in Addis Ababa, Ethiopia. Integr Pharm Res Pract. 2020; 9: 105-112. [CrossRef]

[12] Bahlol M, Dewey RS. Pandemic preparedness of community pharmacies for COVID-19. Res Social Adm Pharm. 2021; 17(1): 1888-1896. [CrossRef]

[13] Zaidi STR, Hasan SS. Personal protective practices and pharmacy services delivery by community pharmacists during COVID-19 pandemic: Results from a national survey. Res Social Adm Pharm. 2021; 17(1): 1832-1837. [CrossRef]

[14] Sum ZZ, Ow CJ. Community pharmacy response to infection control during COVID-19. A cross-sectional survey. Res Social Adm Pharm. 2021; 17(1): 1845-1852. [CrossRef]

[15] Dzingirai B, Matyanga C, Mudzviti T, Siyawamwaya M, Tagwireyi D. Risks to the community pharmacists and pharmacy personnel during COVID-19 pandemic: perspectives from a low-income country. J Pharm Policy Pract. 2020; 13(1): 1-6. [CrossRef]

[16] Hoti K, Jakupi A, Hetemi D, Raka D, Hughes J, Desselle S. Provision of community pharmacy services during COVID-19 pandemic: a cross sectional study of community pharmacists' experiences with preventative measures and sources of information. Int J Clin Pharm. 2020; 42(4): 1197-1206. [CrossRef]

[17] El-Hage W, Hingray C, Lemogne C, Yrondi A, Brunault P, Bienvenu T, et al. Health professionals facing the coronavirus disease 2019 (COVID-19) pandemic: What are the mental health risks? Encephale. 2020: 73-80. [CrossRef]

[18] Ashiru-Oredope D, Chan AHY, Olaoye O, Rutter V. Needs assessment and impact of COVID-19 on pharmacy professionals in 31 commonwealth countries. J Pharm Policy Pract. 2020;13(1): 1-11. [CrossRef]

[19] Lange M, Joo S, Couette P-A, De Jaegher S, Joly F, Humbert X. Impact on mental health of the COVID-19 outbreak among community pharmacists during the sanitary lockdown period. Ann Pharm Fr. 2020;78(6):459-463. [CrossRef]

[20] Johnson A, Vincent B, Carson P, Skoy E. Prevalence of SARS-CoV-2 antibodies among North Dakota community pharmacy personnel: A seroprevalence survey. J Am Pharm Assoc. 2021: 127-132. [CrossRef]

[21] Strand MA, Bratberg J, Eukel H, Hardy M, Williams C. Peer Reviewed: Community Pharmacists' Contributions to Disease Management During the COVID-19 Pandemic. Prev Chronic Dis. 2020; 17: 1-8 [CrossRef]

[22] Cerbin-Koczorowska M, Waszyk-Nowaczyk M, Przymuszała P. Pharmacists' Preparedness to Patients Education at the Time of Pandemic - A Cross-Sectional Study with an Example of SARS-CoV-2 Outbreak in Poland. Int J Environ Res Public Health. 2020; 17(18): 6659. [CrossRef]

This is an open access article which is publicly available on our journal's website under Institutional Repository at http://dspace.marmara.edu.tr. 